\title{
Provando e Riprovando: Galilean science in times of virulence
}

\author{
Vincenzo Aquilanti ${ }^{1}$
}

Published online: 25 May 2020 / Published online: 26 May 2020

(c) Accademia Nazionale dei Lincei 2020

«The aim of science is not to open a door to infinite wisdom, but to set a limit to infinite error»—-says Galileo to his daughter Virginia whilst continuing to do research and teaching under house arrest [Life of Galileo, Ninth Scene]. Bertolt Brecht wrote his play while in exile from Germany at the outbreak of disrupting World War II. This Editorial for the Journal Rendiconti Lincei, Scienze Fisiche e Naturali, appears in the spring of the year 2020, severely affected by the devastating COVID-19 pandemic, while most of our authors and readers are unable to access laboratories and classrooms and have to meet colleagues and students telematically from home.

The Lincei Academy was founded in Rome in 1603 and Galileo was affiliated in 1612. From then on, he added Linceo to his name in his publications: the cover of the present issue reproduces the frontispiece and a remarkable inner page of the Dialogue, by Galileo Galilei Linceo-the celebrated 1632 book where he compares the Ptolemaic and the Copernican systems.

Under the present circumstances, a main modus operandi of the Academy, hosting lively meetings, discussion groups, lectures, and large conferences, has to be radically reconsidered. President Giorgio Parisi, forced to temporarily close the gates, remarks that however «the Academy retains all the competences required to face the many-sided issues connected with this health emergency. Our Statute clearly states that the Academy aims at promoting, coordinating, integrating and spreading scientific knowledge at its highest level, against the background of the unity and universality of culture. Moreover, one of the Academy's tasks is to provide - on demand or upon its own initiative-opinions to public authorities in the fields of its competence and possibly formulate proposals. The Lincei Academy has therefore the duty of ensuring that scientific competence $[\ldots]$ is

Vincenzo Aquilanti

vincenzoaquilanti@yahoo.it

1 Dipartimento di Chimica, Biologia e Biotecnologie, Università di Perugia, Via Elce di Sotto 8, 06123 Perugia, Italy at the service of public opinion and public authorities to allow them to organise their ideas, especially before making decisions that have to be founded on a scrupulous analysis of knowledge. The Academy's evaluations should also-if not primarily-address controversial issues, especially those about which society calls for reliable information in order to make decisions. In this case, scientific progress is achieved by probing the advantages and the faults of the various theses, "provando e riprovando", approving and disproving, in the wake of our great fellow Galileo Galilei. The Lincei Academy has the duty to intervene in the public and scientific debate, in depth and rapidly, in this situation in which taking prompt and effective measures is mandatory. The need to combine skills of so varied a nature suggests going beyond the Academy's study committees that had originally been envisioned in a different perspective. Our Board of Directors has therefore appointed an ad hoc committee [...] with the task of addressing the full range of problems related to COVID-19...»: the Academy collects documentation on resources of this and all other committees, and on contributions by members in various forms, that can specifically be retrieved on the website of the Academy (https://www. lincei.it/it) and in a regularly published newsletter-the one appearing at the time of writing contains the latest documents on facing the pandemic, its impact on the economy and employment, and the role of Europe.

Although the mantra "provando e riprovando" is correctly considered as paradigmatic of Galilean science, it can be traced back to Medieval times: it appears in the Divine Comedy [Third Canticle, Third Canto, Third Verse] used by Dante in the early fourteenth century with reference to the search for beauty and truth in the "divine". When adapted to the permanent dilemma of editors of scientific journals, it would be read as the accept vs reject decision on a submitted paper.

The system of reviewing by anonymous peers may not be perfect, but provides reasonable guarantees in the common practice of the international community of modern scientists. In these times, the attack by false theories can be virulent, and fake news of miraculous recipes permeate the 
media: a scientific journal must stand on the watch tower, receiving submissions of full papers, or drafts as proposals for manuscripts. It is curious to read in an editorial appearing simultaneously in more than 50 journals of the authoritative American Chemical Society https://pubs.acs. org/doi/10.1021/jacs.0c04253. «Throughout this period, we seek to assist our authors, reviewers, and readers, despite the exceptional disruption to their normal research workflow. One area of particular concern is that authors may require extra time to prepare revised manuscripts, especially if additional laboratory-based experimental work is required to address specific reviewer or editor concerns. At this time, reviewers may consider limiting their recommendations for additional experimental work, except in those cases where they deem it necessary to support the central claims of the paper and maintain high standards of science/ engineering...». It makes sense, but even a small aperture may assist error to enter by the back door.

In 1870, the capital of Italy was set in Rome, the Lincei Academy was re-established and this Journal founded in order to provide the proceedings: so this year we celebrate our 150th anniversary. Thirty years ago, Academician Ernesto Capanna started the present series, acting as chief editor until 2002, when he passed on the baton to colleague Sandro Pignatti, who was responsible for the journal until 2011 (from 2009 Rendiconti Lincei has been separated into two, this journal published by Springer and a companion one dedicated to mathematics). Capanna and Pignatti, both professors in areas of life sciences (zoology and botany, respectively) at the Sapienza University of Rome, and presently Emeriti, have been very active until now as Section Editors. Acknowledging their continuing contributions to the success of the Journal, the decision has been taken to nominate both as Editors-in-Chief Emeriti.

A change regards the position of Deputy Editor-in-Chief. We acknowledge the several years of invaluable service constantly offered in this role by Annibale Mottana, supporting the late Francesco Paolo Sassi, Editor-in-Chief from 2011 to 2016 - and myself since then and until now. Mottana, a former professor of Geosciences at the University of Roma Tre and currently Adjunct Secretary of the Class of Sciences in the Lincei Academy, to my regret expressed his wish to resign, but for a valid reason: he was elected last year as the
President of the Italian National Academy of Sciences (also known as of the XL). This is a very prestigious achievement, for which I offer him the warmest congratulations; however, it is becoming a very demanding position, the more so in these difficult times.

These changes induced some rearrangement and integration in the Editorial Board, some of which are mentioned in the following. To begin with, we are particularly honored to welcome as the new Deputy Editor-in-Chief Giuseppe Orombelli, Emeritus Professor in the University of Milan Bicocca, also from the area of geosciences. Academician Orombelli has been acting for a decade as member of the Editorial Board of the Journal in the role of Section Editor, and also as editor of several special collections of papers in the environmental area. In the present issue, he contributes with an article belonging to a Topical Collection on Coastal Protection that he co-edited.

The content of this issue features articles belonging to several other Topical Collections, which are principally put forward by Academicians and originated mainly from events organized by them. Therefore it is exemplary of the new editorial policy that we aim at reducing unsolicited submissions and give priority to the dissemination of the scientific progress fostered by the Academy and its members through a variety of initiatives in the Rome premises and elsewhere. Among the Topical Collections being continued and still open, we mention the one on Anisotropic Properties of Matter, co-edited by the geoscientist Giovanni Ferraris, and another one on Classical and Quantum Plasmas, co-edited by physicist Francesco Pegoraro. A comprehensive Special Issue of the journal, edited by astronomer and astrophysicist Marco Tavani, is published separately and accounted for in the above-mentioned newsletter. Ferraris, Pegoraro and Tavani join the Board as Section Editors from this issue: we also welcome in this role, reserved to Academicians, Paola Bonfante, biologist, and Francesco Berrilli, geoscientist.

Vincenzo Aquilanti, Linceo

Editor-in-Chief

Publisher's Note Springer Nature remains neutral with regard to jurisdictional claims in published maps and institutional affiliations. 